\title{
Erratum
}

Diabetologe 2019 $\cdot 15: 478$

https://doi.org/10.1007/s11428-019-0501-9

Online publiziert: 26. Juni 2019

(c) Springer Medizin Verlag GmbH, ein Teil von Springer Nature 2019

\section{Sabine Kahl}

Institut für klinische Diabetologie, Deutsches Diabetes-Zentrum, Heinrich-Heine-Universität, LeibnizZentrum für Diabetesforschung, Düsseldorf, Deutschland

\section{Erratum zu: Frühzeitige Kombinationstherapie bei Typ-2-Diabetes}

\section{Erratum zu:}

Der Diabetologe 2019

https://doi.org/10.1007/s11428-0190452-1

In diesem Beitrag bitten wir darum, folgende präzisierten Angaben bezüglich der Darstellung des Ausgangs- $\mathrm{HbA}_{1 \mathrm{c}}$ $\left(\mathrm{HbA}_{1 \mathrm{c}}\right.$ : Glykohämoglobin Typ $\left.\mathrm{A}_{1 \mathrm{c}}\right)$ einer Studiensubgruppe zu beachten:

„Der Anteil von Studienteilnehmern mit einem $\mathrm{HbA}_{1 c}<7,0 \%$ nach 20 Wochen war mit Sitagliptin größer als mit Plazebo im gesamten Studienkollektiv (28,8\% vs. $16,6 \%, p=0,002)$ sowie in der Subgruppe mit einem Ausgangs- $H b A_{1 c}$ von $\geq 8,5 \%$ (15,6\% vs. $5,7 \%, \boldsymbol{p}=\mathbf{0 , 0 2 6})$.

Des Weiteren bitten wir Sie, folgende Umformulierungen bezüglich der Beschreibung der Sicherheit der Behandlungen und der Therapieintensivierungsstrategien zu beachten:

„In Bezug auf die Sicherheit bei den Behandlungen gab es keine statistisch signifikanten Unterschiede zwischen den Gruppen hinsichtlich der Häufigkeit (schwerer) unerwünschter Ereignisse, $\mathrm{Hy}$ poglykämien, Gewichtsänderung oder der weiteren Sicherheitsparameter (z. B. Blutdruck, Herzfrequenz, Laborwerte)."

„Allgemein gut verträgliche, effektive und geeignete Therapieintensivierungsstrategien könnten dazu beitragen, diese Verzögerung in der notwendigen Therapieanpassung der Patienten zu verringern."

\section{Korrespondenzadresse}

\section{Dr. Sabine Kahl}

Institut für klinische Diabetologie, Deutsches Diabetes-Zentrum, Heinrich-Heine-Universität, Leibniz-Zentrum für Diabetesforschung Auf'm Hennekamp 65, 40225 Düsseldorf, Deutschland

Sabine.Kahl@ddz.de 